\title{
EQUIVARIANT MAPS WHICH ARE SELF HOMOTOPY EQUIVALENCES ${ }^{1}$
}

\author{
E. DROR, W. G. DWYER AND D. M. KAN
}

\begin{abstract}
The aim of this note is (i) to give (in 82) a precise statement and proof of the (to some extent well-known) fact that the most elementary homotopy theory of "simplicial sets on which a fixed simplicial group $H$ acts" is equivalent to the homotopy theory of "simplicial sets over the classifying complex $\bar{W} H$ ", and (ii) to use this (in 81) to prove a classification theorem for simplicial sets with an $\mathrm{H}$-action, which provides classifying complexes for their equivariant maps which are self homotopy equivalences.
\end{abstract}

1. The classification theorem. In order to formulate our classification theorem (1.2) we need some definitions involving

1.1. Simplicial sets With a Simplicial group action. (i) Let $V$ and $V^{\prime}$ be simplicial sets on which a simplicial group $H$ acts (from the right). A map $V \rightarrow V^{\prime}$ then will be called an equivariant weak homotopy equivalence if it is compatible with the action of $H$ and is a weak homotopy equivalence. This should not be confused with the stronger notion of weak equivariant-homotopy equivalence, which is a map compatible with the action of $H$, which induces weak homotopy equivalences on the fixed point sets of all simplicial subgroups of $H$ (and not merely the identity subgroup).

(ii) Let $V$ be a simplicial set on which a simplicial group $H$ acts. Then we denote by haut ${ }_{H} V$ the simplicial monoid of equivariant weak self homotopy equivalences of $V$, i.e., the simplicial monoid which has as $n$-simplices the commutative diagrams

$$
\begin{array}{ccc}
\Delta[n] \times V & \rightarrow & \Delta[n] \times V \\
\text { proj. } \searrow & & \swarrow \text { proj. } \\
& \Delta[n] &
\end{array}
$$

in which the top map is an equivariant weak homotopy equivalence.

1.2. Classification theOREM. Let $\boldsymbol{H}$ be a simplicial group and $W H$ its classifying complex $[4, p$. 87] and let $M$ be a minimal simplicial set $[4, p .35]$ and aut $M$ its simplicial group of automorphisms [1, 1.3], [4, p. 74]. Then the function complex $(\bar{W} \text { aut } M)^{\bar{W} H}$ has the following properties:

(i) The components of ( $\bar{W}$ aut $M)^{\bar{W} H}$ are in natural 1-1 correspondence with the equivariant weak homotopy equivalence classes of simplicial sets with an $\mathrm{H}$-action, which have the weak homotopy type of M. Moreover each such class contains

Received by the editors October 31, 1979 and, in revised form, January 25, 1980.

1980 Mathematics Subject Classification. Primary 55P15.

${ }^{1}$ This research was supported in part by the National Science Foundation and the Israeli Academy of Sciences. 
simplicial sets which satisfy the extension condition $[4, p .2]$ and on which the $H$-action is free (i.e. principal $[4, p .70]$ ).

(ii) If $V$ is a simplicial set which satisfies the extension condition and has the homotopy type of $M$ and on which $H$ acts freely, then $\bar{W}$ haut $_{H} V$ has the weak homotopy type of the component of $(\bar{W} \text { aut } M)^{\overline{W H}}$ which (see (i)) corresponds to $V$.

Proof. This is an immediate consequence of the classification theorem $[1,1.4]$ for fibrations over $\bar{W} H$ and the results of $\$ 3$.

1.3. Corollary. Let $H$ be a simplicial group and let $G$ be a group which has no center and no outer automorphisms (such as, for instance, the symmetric group on $n$ letters, where $n \neq 2$ or 6$)$. Then

(i) there is only one equivariant weak homotopy equivalence class of simplicial sets with an $H$-action, which have the weak homotopy type of $K(G, 1)$, and

(ii) if $V$ is a simplicial set which satisfies the extension condition and has the homotopy type of $K(G, 1)$ and on which $H$ acts freely, then haut $_{H} V$ is contractible (i.e. has the weak homotopy type of a point).

Proof. This follows immediately from the fact that aut $K(G, 1)$ is contractible.

1.4. Corollary. If $V$ is a contractible simplicial set which satisfies the extension condition and on which a simplicial group $H$ acts freely, then haut $_{H} V$ is contractible.

2. The equivalence of homotopy theories. Let $H$ be a simplicial group, let $\mathbf{S}_{H}$ denote the category of simplicial sets with a (right) $\boldsymbol{H}$-action and equivariant maps between them, and let $\mathbf{S} / \bar{W} H$ be the category of simplicial sets over the classifying complex [4, p. 87] $\bar{W} H$. Then the homotopy theories of $\mathbf{S}_{H}$ and $\mathbf{S} / \bar{W} H$ are equivalent in the sense that Theorem 2.1 and Corollary 2.5 below hold.

2.1. TheOREM. The simplicial localizations [3, 83] of $\mathbf{S}_{H}$ with respect to the equivariant weak homotopy equivalences (1.1) and of $\mathbf{S} / \bar{W} H$ with respect to the weak homotopy equivalences over $\bar{W} H$ are simplicial categories in the sense of $[3, \S 2]$, which are homotopically equivalent $[3,2.5]$.

This follows immediately from $[2,3.5],[3,4.1]$ and the following propositions.

2.2. Proposition. The categories $\mathbf{S} / \bar{W} H$ and $\mathbf{S}_{H}$, with fibrations, cofibrations and weak equivalences as defined below, are closed model categories in the sense of Quillen [5]:

(i) The model category structure on $\mathrm{S} / \bar{W} H$ is the one induced by the usual one on the category of simplicial sets S [5, II, 2.8]; in particular the weak equivalences are the weak homotopy equivalences over $\bar{W} H$.

(ii) $A$ map in $\mathbf{S}_{H}$ is a fibration if the underlying map in $\mathbf{S}$ is so, is a cofibration if it is 1-1 and its $H$-action is relatively free (i.e. no nonidentity simplex of $H$ fixes $a$ simplex of the range which is not in the image of the domain), and is a weak equivalence if it is an equivariant weak homotopy equivalence (1.1).

The proof is straightforward. 
2.3. Proposition. There is a pair of adjoint functors $A: \mathbf{S} / \bar{W} H \rightarrow \mathbf{S}_{H}$ (the left adjoint) and $B: S_{H} \rightarrow \mathrm{S} / \bar{W} H$ (the right adjoint) such that, in the terminology of 2.2

(i) both functors send weak equivalences into weak equivalences, and

(ii) for every object $U \in \mathrm{S} / \bar{W} H$ and every object $V \in \mathbf{S}_{H}$, the adjunction maps $U \rightarrow B A U$ and $A B V \rightarrow V$ are weak equivalences.

Proof. Given an object $U: Y \rightarrow \bar{W} H \in S / \bar{W} H$, one defines $A U$ by $[4,821]$ $A U=Y \times \times_{\bar{W} H} W H$, with its $H$-action induced by the one on $W H$ and, given an object $V \in \mathbf{S}_{H}$, one defines $B V$ as the map $(V \times W H) / H \rightarrow \bar{W} H \in \mathbf{S}$ induced by the projection $V \times W H \rightarrow W H \in \mathbf{S}_{H}$. The adjoint of a map $f: A U \rightarrow V \in \mathbf{S}_{H}$ is the map $Y \approx A U / H \rightarrow(V \times W H) / H \in \mathrm{S}$ over $\bar{W} H$ induced by the map $A U \rightarrow V \times W H \in \mathbf{S}_{H}$ which is the product of $f$ and the obvious map $A U=$ $Y \times_{\bar{W} H} W H \rightarrow W H \in \mathbf{S}_{H}$. The rest of the proof is a straightforward verification.

Also not hard to verify is

2.4. Proposition. Let $(\mathrm{S} / \bar{W} H)_{*}$ and $\left(\mathrm{S}_{H}\right)_{*}$ denote the simplicial categories $[3,82]$ obtained from the model categories $\mathrm{S} / \bar{W} H$ and $\mathbf{S}_{H}(2.2)$ by giving them the obvious simplicial structure (i.e. function complexes). Then $(\mathrm{S} / \bar{W} H)_{*}$ and $\left(\mathbf{S}_{H}\right)_{*}$ are closed simplicial model categories in the sense of Quillen [5]. Moreover, the functors $A$ and $B$ of 2.3 induce functors $A_{*}:(\mathbf{S} / \bar{W} H)_{*} \rightarrow\left(\mathbf{S}_{H}\right)_{*}$ and $B_{*}:\left(\mathbf{S}_{H}\right)_{*} \rightarrow(\mathbf{S} / \bar{W} H)_{*}$.

Let $(\mathbf{S} / \bar{W} H)_{*}^{\text {cf }} \subset(\mathbf{S} / \bar{W} H)_{*}$ and $\left(\mathbf{S}_{H}\right)_{*}^{\text {cf }} \subset\left(\mathbf{S}_{H}\right)_{*}$ denote the subcategories generated by the objects which are cofibrant as well as fibrant (i.e. the fibrations with $\bar{W} H$ as base and the fibrant simplicial sets with free $H$-actions). Then the functors $A_{*}$ and $B_{*}(2.4)$ send these simplicial subcategories into each other and one has, in view of $[3,4.8]$ :

2.5. Corollary. The simplicial categories $(\mathrm{S} / \bar{W} H)_{*}^{\mathrm{cf}}$ and $\left(\mathbf{S}_{H}\right)_{*}^{\mathrm{cf}}$ are homotopically equivalent [3, 2.5]. Moreover, they are weakly equivalent $[3,2.4]$ to the simplicial categories of Theorem 2.1 .

\section{BIBLIOGRAPHY}

1. E. Dror, W. G. Dwyer and D. M. Kan, Automorphisms of fibrations, Proc. Amer. Math. Soc. 80 (1980), 491-494.

2. W. G. Dwyer and D. M. Kan, Calculating simplicial localizations, J. Pure Appl. Algebra (to appear).

3.

4. J. P. May, Simplicial objects in algebraic topology, Van Nostrand, Princeton, N.J., 1967.

5. D. G. Quillen, Homotopical algebra, Lecture Notes in Math., vol. 43, Springer-Verlag, New York, 1967.

Department of Mathematics, Hebrew University, Jerusalem, Israel

Department of Mathematics, Yale Universtty, New Haven, Connecticut 06520

Department of Mathematics, Massachusetts institute of Technology, Cambridoe, MasSACHUSETTS 02139 